\title{
Autism as a Natural Human Variation: Reflections on the Claims of the Neurodiversity Movement
}

Pier Jaarsma and Stellan Welin

\section{Linköping University Post Print}

N.B.: When citing this work, cite the original article.

The original publication is available at www.springerlink.com:

Pier Jaarsma and Stellan Welin, Autism as a Natural Human Variation: Reflections on the Claims of the Neurodiversity Movement, 2012, Health Care Analysis, (20), 1, 20-30.

http://dx.doi.org/10.1007/s10728-011-0169-9

Copyright: Springer Verlag (Germany) http://www.springerlink.com/

Postprint available at: Linköping University Electronic Press http://urn.kb.se/resolve?urn=urn:nbn:se:liu:diva-72172 
1 Abstract Neurodiversity has remained a controversial concept over the last decade. In its 2 broadest sense the concept of neurodiversity regards atypical neurological development as a normal human difference. The neurodiversity claim contains at least two different aspects.

4 The first aspect is that autism, among other neurological conditions, is first and foremost a natural variation. The other aspect is about conferring rights and in particular value to the neurodiversity condition, demanding recognition and acceptance. Autism can be seen as a natural variation on par with for example homosexuality. The broad version of the neurodiversity claim, covering low-functioning as well as high-functioning autism, is problematic. Only a narrow conception of neurodiversity, referring exclusively to highfunctioning autists, is reasonable. We will discuss the effects of DSM categorization and the medical model for high functioning autists. After a discussion of autism as a culture we will analyze various possible strategies for the neurodiversity movement to claim extra resources for autists as members of an underprivileged culture without being labelled disabled or as having a disorder. We will discuss their vulnerable status as a group and what obligation that confers on the majority of neurotypicals.

Keywords Autism - Disability - DSM-V - Equality - Neurodiversity - Vulnerability

\section{Introduction}

In this paper we will discuss some issues around autism, in particular the neurodiversity claim proposed by some autists. What we call the neurodiversity claim consists of at least two parts. One is related to the idea that there are indeed neurological (or brain-wiring) differences among the human population. Being autistic is one of them. One aspect of the neurodiversity claim is that autism (or some other neurological condition) is a natural variation among humans. Being neurodiverse or neurotypical ("normal") are just different ways of existing as humans. discrimination and other more political issues. The two aspects often go together. There now exists what might be called a neurodiversity movement. The term 'neurodiversity' is generally credited to Judy Singer, a sociologist diagnosed with Asperger Syndrome [22, 26]. The neurodiversity movement was developed in the 1990s by online groups of (high-functioning) autistic persons $[12,22]$. It is now associated with the struggle for the civil rights of all those 
diagnosed with neurological or neurodevelopmental disorders, such as attention deficithyperactivity disorder, bipolar disorder, developmental dyspraxia, dyslexia, epilepsy, and Tourette's syndrome [8]. Neurodiversity has remained a controversial concept over the last decade. In this paper, we will limit ourselves to neurodiversity as it relates to autism. We choose this because there is a greater chance of clarifying the moral problems surrounding neurodiversity for one particular condition then there is for a set of fairly disparate conditions.

One moral issue concerns the usage of terms. 'Person with autism' suggests that there is a normal person trapped behind the autism. It carries with it the idea that a person is somehow separable from autism. But this term is met with opposition from the neurodiversity movement. They claim that autism is inseparable from the person and being autistic influences every single element of who a person is. [29] The autistic autobiographical author Temple Grandin seems to hold this view: "If I could snap my fingers and be nonautistic, I would not. Autism is part of what I am" [11:xviii]. Therefore the neurodiversity movement prefers to speak of 'autistic persons' or 'autists' rather than 'persons with autism'. Donna Williams, another autistic autobiographical author, seems to hold the opposite view. She refers to her autism poetically as "an invisible prison with replica selves on the outside, each a contortion, a distortion of the one you can't see, who can't get out" [27:9].

We will in many places distinguish between "high-functioning autists" and "low-functioning autists". There seems to be a partial consensus on this distinction: if autists have an IQ in the normal range (or above), they usually are said to have high-functioning autism (HFA) [3].

Our aim in this paper is to understand the neurodiversity claim - or rather claims - and to analyze them and relate them to other discussions. To do this we will first describe autism. Secondly, we will discuss the claim(s) of neurodiversity and we will distinguish between a narrow and a broad view on neurodiversity. Thirdly, we will discuss the effects of DSM categorization and the medical model for high functioning autists. Fourthly, we will discuss autism as a culture. In this last section we will also analyze various possible strategies for the neurodiversity movement to claim extra resources as a group without being labelled disabled or as having a disorder. We will discuss their vulnerable status as a group and what obligation that confers on the majority of neurotypicals.

\section{Autism}


Autism was first identified by Leo Kanner in 1943 as a childhood syndrome characterized by 'autistic aloneness,' obsession with routine and profound problems with communication. Asperger's Syndrome (AS), first described by Hans Asperger in 1944, differs from 'classic' autism in that those diagnosed with Asperger's do not show evidence of intellectual deficiency or language delay [7]. The concept of the autism spectrum disorder (ASD), Asperger's disorder at the mild end to severe autistic disorder at the other end, was developed in the early 1990s by Lorna Wing [28]. The primary diagnostic abnormalities of autism involve selective impairments in social, communicative and imaginative abilities that are usually quite severe. About seventy-five percent of diagnosed autists have intellectual disabilities, the rest have average to good IQs [18]. Sensory difficulties are also quite common in ASD. These difficulties may be connected to sound and hearing, sight and seeing, touch, taste or general sensory dysphoria [16]

Much of the literature in the 1940s speculated that autism was associated in particular with emotionally frigid mothers, who became known as "refrigerator mothers" [14]. The increasing importance of cognitive science, brain science and then biogenetic science, investigations into the biological and genetic basis for autism, eventually removed the association between autism and parenting [7].

Numerous psychological, biological and neurological theories have been proposed to explain autism. These theories of autism share the assumption that there is a deficit in autists that should be researched, classified, and, ultimately, modified. Therefore, the common link among these theories is an assumption that there is something wrong with the person with autism [5].

In general, in the scientific community, autism is not regarded as a single disease but as a syndrome with multiple nongenetic and genetic causes [20]. Moy, a molecular psychiatry researcher, states that the etiology of autism is thought to involve an interaction between genetic susceptibility, mediated by multiple genes, and possible environmental factors, leading to aberrant neurodevelopment [19]. In a recent review article it is stated that autism spectrum disorder is highly genetic [17]. The relative risk of a second child having this diagnosis is $20-50$ times higher than the population base rate if the first child is affected. Heritability estimates from family and twin studies suggest that about $90 \%$ of variance is attributable to genetic factors, making this disorder the neuropsychiatric disorder most affected by genetic factors.

At present Autistic Disorder and Asperger's Disorder are two separate diagnoses included in the Diagnostic and Statistical Manual of Mental Disorders-IV TR (DSM-IV TR). 
103 The latest development at the time of writing this paper is that the work group of DSM-V

104 proposes Asperger's disorder to be subsumed into an existing disorder: Autistic Disorder 105 (Autism Spectrum Disorder) [33].

In our opinion, high-functioning autism should neither be regarded as a disorder or a disability nor as an undesirable condition per se, but rather as a condition with a particular vulnerability. Autism can also have desirable and enabling consequences, both to the individual and to society. Dr. Temple Grandin refers to this when she speaks about the origin of her own success:

\section{Neurodiversity}

Judy Singer [22, 26] argued for a politics of "neurodiversity," asserting that "[t]he 'neurologically different' represent a new addition to the familiar political categories of class/gender/race". Sparked by this first articulation of neurodiversity the bush fire of neurodiversity quickly spread across the internet. As a consequence different conceptions of neurodiversity emerged in the past decade. These can be roughly divided into broad and narrow conceptions.

In its broadest sense the concept of neurodiversity defines all atypical neurological development as a normal human difference that should be tolerated and respected in the same way as other human differences [12]. In slightly different ways a number of authors $[2,4,8]$ suggest that people with different neurological conditions are just different, not handicapped or pathological.

One conception, referring only to autism, is suggested by Baker [2]. She states that in the case of autism and other neurological differences, the former is called neurodiversity and the latter is neurological disability. Proponents of the neurodiversity movement claim their condition is not something to be cured, but rather a human specificity 
or difference, with different ways of socializing, communicating and sensing, that may not necessarily be disadvantageous and that must be equally respected [22].

Brain structure and neurological development figure prominently in some of the descriptions of neurodiversity on the internet: "Neurodiversity is the idea that variation in brain development and function should be appreciated and accepted as any other form of physical variation." [30] And: "Neurodiversity is defined as the whole of human mental or psychological neurological structures or behaviors, seen as not necessarily problematic, but as alternate, acceptable forms of human biology." [31]

The moral and political aspects of neurodiversity are also emphasized. "Neurodiversity, in its broadest usage, is a philosophy of social acceptance and equal opportunity for all individuals regardless of their neurology."[32] Essentially, from a moral and political point differences in brain structure and neurological functioning are claimed to have no more significance than differences in skin colour or sex.

As outlined above the neurodiversity claim contains at least two different aspects. One aspect is that autism (or at least high-functioning autism) is not to be treated like a disability or a handicap but rather as a natural variation. The other aspect is about conferring rights and in particular value to the neurodiversity condition, perhaps again to highfunctioning autism. This condition is not just natural and not pathological, but also valuable. The political claim of the neurodiversity movement goes beyond just giving rights to autistic persons similar to rights to handicapped people; it also claims for recognition and acceptance. We will start with discussing autism as a natural variation and leave the moral and political claims to a later section.

\section{Natural variation}

The first point to be made is obviously that "normal variation" is a concept that can be understood both in a statistical sense (how common is it?) but also in an evaluative sense (how normal is it?). A typical example of the statistical sense is that there is a certain gene variety with a certain frequency in the human gene pool. Such a gene can be connected to diseases or to more desirable traits. From an evolutionary point of view such a gene being part of the normal (statistical) variation should not just be caused by a mutation (it may of course have started its existence in this way). Its survival in the human gene pool should have some evolutionary explanation; basically that it has some beneficial effects. One example is Sickle 
170 Cell Anemia, where having one allele of the genes gives enhanced resistance to malaria;

171 having two alleles causes Sickle cell anemia. The gene survives in the human gene pool 172 because having one allele is good for survival and propagation even if two alleles are harmful.

173 If autism is a normal variation in the statistical sense, there should be some evolutionary 174 explanation of its continued existence.

175 If autism is caused by environmental factors during the fetal time, it is not a 176 natural variation in this particular sense. It is then caused by something other than a specific 177 gene, more like a harm that happened. If it had been true that autism was connected to 178 vaccinations, then autism could not be a natural variation. (If only a part of autism was caused 179 in that way, at least that part could not be a natural variation.) If however autism is caused 180 randomly during the fetal development of the brain, it could still be seen as a natural 181 variation, namely if such random processes are naturally occurring without any clear outside 182 cause. A possibility is also a combination; there may be a combination of genes that makes 183 the brain susceptible to certain random changes etc. All these variants can be part of normal 184 variations.

\section{Effects of DSM classification}

The DSM classification is a typical example of the so-called 'medical model' of interpreting behavior. According to this model individuals are disabled because of their deficits and 191 difficulties, which are summed up in DSM-IV TR (see e.g. Diagnostic criteria for 299.00 192 Autistic Disorder and Diagnostic criteria for 299.80 Asperger's Disorder). The focus, in the medical model, is upon fixing, curing and correcting deficits and difficulties to enable the individual to live in normal society [16]. Neurodiversity movement adherents have protested against the medicalisation of the condition now known as Asperger's Disorder. Sarah Allred suggests that they should take as an example a successful precedent: the gay rights movement [1]. The American Psychiatric Association (1973) declared that homosexuality per se was no

198 longer a psychiatric disorder. The philosopher Lennart Nordenfelt explains the rationale behind this significant change [21]. Homosexuality was no longer seen as a disorder because it did not regularly cause subjective distress or was associated with general impairment in social effectiveness or functioning. In the new outlook on the general concept of a mental disorder it was argued that the consequences of a condition, and not its etiology, determined whether the condition should be considered as a disorder. 
An important observation regarding homosexuality is this. In a society with strong prejudice of homosexuality the lives of homosexuals will be troubled. They will be afraid to be open about it and try to pass as heterosexuals. Homosexuals will be unhappy and will have many psychological and psychiatric problems caused not by their sexual preferences but by society. In a homophobic society nearly all homosexuals will appear pathological. The cure for these problems has simply been a wider acceptance of homosexuality. We should expect that many autists in a similar way have psychiatric and psychological problems due to the "autism-phobic" character of present society. In similarity with homosexuals most of the problems for high-functioning autists may be due to social conditions. To say that these people have a mental disorder because of the consequences of their condition is in a sense blaming the victim. The consequences of their condition are perhaps for a very important part the result of society's reaction to their condition. Nordenfelt wrote that much of the distress of homosexuals can be explained in terms of severe circumstances rather than in terms of inner constitution [21]. It is our belief that the same, mutatis mutandis, can be said of highfunctioning autists.

To subsume Asperger's Disorder into Autistic disorder in DSM-V is a wrong way to go. To be put in the same category together with low-functioning autists may be regarded by some of the persons with Asperger's as an even worse stigmatization.

\section{Autism as a culture}

Is there a specific autistic culture? Dawn Prince-Hughes, an Asperger Syndrome autobiographical writer, believes there is: “[m] uch like the Deaf community, we autists are building an emergent culture. We individuals, with our cultures of one, are building a culture of many" [6:793]. Joyce Davidson notices distinctive autistic styles of communication particularly online, which she calls Wittgensteinian 'language games'. There is a parallel between the 'language games' of deaf people and those of autistic people in that both populations have a communication style that is different from the norm [6].

Davidson calls the autistic culture a 'minoritized' culture, referring to

234 discrimination and exclusion, comparable to Queer, Black or Deaf cultures. The internet, 235 however, has given autists the means to find a way around social and communicative 236 exclusion, "[f]reed from the constraints of NT [neurotypical] timing, NTways of interpreting 237 body language, free from the information overwhelm of eye contact, the energy demands of 
managing body language" [6:801]. Many of those with autism are using the internet to connect with similar others, binding them together, somewhat paradoxically, into groups. The new virtual environment is much more autism compatible than the regular social environment, which has become more and more autism incompatible during the last centuries [6].

Using the internet in a particular way seems to be a solution for some of the psychosocial problems high-functioning autists encounter. More communication, mutual support, group bonding, even the creation of an autistic culture, all these have become possible for autists because of the autism-compatible features of the internet. The internet, though, should be seen only as a way of facilitating communication, not as constituting a specific culture in itself. The claim that there is a specific autistic culture can be based on autistic persons difference in language style, ways of relating to others, values etc. Above all is the common interest that they share, similar to most other cultural or ethnic groups. Typical for such groups are that their members want to identify with the group.

One of the possible strategies for the neurodiversity movement is to have autists (or at least high-functioning autists) recognized as a special group in need of certain "group rights" similar to what has been conferred on various minority groups. The core of such claims is often that there is something special to be protected, for example a certain culture in risk of being swallowed by the majority culture.

They can, like other minority groups, base their claim for special treatment on the pervasive discrimination against them, both historically and also present. The Inuit in Canada, Native Americans in the USA, and Sami population in the Scandinavia are examples of groups that claim special rights. In their case this is based on a common origin and a shared history. This is not the case for autistic people, nor do they have a homeland of their own. The claims from the autistic culture are similar to the Deaf culture, which also live dispersed among the majority. But how to determine whether somebody is a member of the autistic culture? Is it enough with self identification as autistic or do we need some "objective" way to characterize them? In the case of Sami people or Inuit there are "objective" ways; each member shares a common history and background. Although neither autistic people nor Deaf people have a common origin it seems that there are rather simple behavioral traits that together with self-identification will single out members of such a culture.

The autistic culture may benefit from making use of the same philosophical foundations to argue for their minority group rights that the philosopher Will Kymlicka described for ethnocultural minority group rights. Group-specific rights for minorities are needed to ensure that all citizens are treated with genuine equality. On this view, "the 
accommodation of differences is the essence of true equality" [15:108], and group-specific rights are needed to accommodate our differences.

When we apply Kymlicka's thoughts to the autistic culture, we can say that the autistic culture is unfairly disadvantaged in the cultural market-place. Political recognition and support can rectify this disadvantage. The viability of the autistic culture may be undermined by economic and political decisions made by the majority of neurotypicals. The members of the majority culture of neurotypicals do not face this problem. Given the importance of cultural membership, this is a significant inequality which, if not addressed, becomes a serious injustice.

What if the environment of autists is not yet autism-compatible? The environment would be autism-compatible when the society they live in would have adopted the so-called 'social model of disability'. This model suggests that the society is equally responsible for enabling individuals with disabilities to live and exist within the society as disabled people. According to this model we should not want to change the individual so much as accommodate that person and support him or her in ways that enable them to live positively [16]. While most cultures, including the Deaf culture, usually are able to manage on their own, this is not quite clear for the autistic culture. Or rather, one needs a restrictive view of the autistic culture, only including high-functioning autists, to get a potentially independent culture. So, first let us discuss autistic culture from the broad conception, which we deem problematic, and later turn to the more plausible narrow conception.

\section{Autistic vulnerability}

Considering the broad conception of neurodiversity, a paradox becomes clear. If neurodiversity is accepted by society as a special culture, the autists that need care [13] may face a hard time getting it, because their state of being will be regarded as just a natural variation. The high-functioning autists that do not need care live happily in the knowledge that they are freed from the burden of having a deficit and may have a better life with non interference. But it may not be so good for low-functioning autists or even high-functioning autists that $d o$ need care. Acceptance does not 'cure' difficulties with social relationships, social communication, rigidity and sensory issues. On the other hand if neurodiversity is not accepted by society as a separate culture, high-functioning autists will still suffer the stigma of having a deficit, even if some of them do not need special care and support. 
Two strategies can be used to get around this paradox. The first strategy is to recognize the vulnerability in general of most autists. The word "vulnerability" is derived from the Latin verb vulnerare, "to wound." To be vulnerable means to face a significant probability of incurring an identifiable harm while substantially lacking ability and/or means to protect oneself [25]. Autists are vulnerable in this sense. The concept of vulnerability is essential to bioethics. The vulnerability of other human beings is the source of our special responsibilities to them [10].

There are arguments against the labeling and categorization of vulnerable individuals and populations [24]. Labeling individuals as 'vulnerable' risks viewing vulnerable individuals as 'others' worthy of pity, a view rarely appreciated. The essence of these arguments is that the label of 'vulnerability' leads to inequality. These arguments are not plausible, as we will try to show. Vulnerability is something we all, autists and non-autists alike, experience in our lives. We all belong to vulnerable populations during some time of our lives. Vulnerability as a concept does not separate a particular group of people from the rest of mankind, unlike the concepts of disability and disorder. Therefore, vulnerability implies equality rather than inequality.

However, it does remain clear that some people are more vulnerable than other people, e.g. infants, the elderly and of course, by their very nature, autists. These particularly vulnerable people may need care and support. Let us briefly stay inside the broad conception of neurodiversity including all autists. Because vulnerabilities are grounds for special responsibilities, losing the diagnosis of 'disorder' by replacing it with a characterization based on 'vulnerability' should at first sight not have any detrimental effect on the protection of people with autism. The discovery and assessment of autistic vulnerabilities can build upon the existing bodies of knowledge about the natural causes of autism and about the consequences for autists of neurotypicals' actions, choices and social conventions [11]. However, applied to the broad conception of neurodiversity this strategy is problematic, for the pragmatic reason of the enormous amount of resistance that needs to be overcome to stop speaking about 'disorder' in the case of high-functioning autists as well as low-functioning 334 autists.

A better strategy to tackle the difficulties that accompany the acceptance of neurodiversity by society is to adhere only to the narrow version of the neurodiversity claim. The reason for this preferred adherence is that the broad version of the neurodiversity claim is problematic. It is clear that people with low-functioning autism are extremely vulnerable and their condition justifies the qualification "disability". However, the degree of social 
construction of their disability has to be taken into account. Members of the group of high341 functioning autists most often can have rather independent lives in the right kind of environment. For what are now disabling traits of these people, may, in a differently constructed social environment, become "neutral" characteristics [23]. Wendy Lawson, autism advocate and scholar, formulates it like this: “[...] only as a society gains understanding of an individual and their cognitive difference ('diff-ability') and also use the understanding to inform appropriate interventions, will that individual's 'disability' be less disabling. " [16: 53] So, disability in autism is always, at least partially, socially constructed disability.

\section{Conclusion}

Some autism inside the narrow conception of neurodiversity can be seen as a natural variation on par with for example homosexuality. (Lower-functioning autism is also part of natural variation but may rightly be viewed as a disability.) Just as homosexuals in a homo-phobic society, the conditions in which autists have to live in an autism-incompatible or even autismphobic society are unreasonable. Therefore, it is not fair to place the locus of the problem solely on the autistic individual. What also is needed is a discourse about the detrimental effects of an autism-incompatible and autism-phobic society on the well-being of autists. Therefore, in the case of high-functioning autists, society should not stigmatize these persons as being disabled, or as having a disorder or use some other deficit-based language to refer to these people. It is much less morally problematic to refer to the particular vulnerability of these autists. Also, group-specific rights for autists are needed to ensure that the autistic culture is treated with genuine equality.

It is our conclusion that it is wrong to subsume all persons with Asperger's Syndrome and high-functioning autists into the wide diagnostic category of Autistic Disorder (Autism Spectrum Disorder), as the work group of the American Psychiatric Association for the Diagnostic and Statistical Manual of Mental Disorders-V (DSM-V) proposes. Some of these persons are not benefited with such a psychiatric defect-based diagnosis. In fact, some of them are being harmed by it, because of the disrespect the diagnosis displays for their natural way of being, which is of course contradictory to the Hippocratic principle of 'primum non nocere'. However, we think that it is still reasonable to include other categories of autism 
in the psychiatric diagnostics. The narrow conception of the neurodiversity claim should be 374 accepted but the broader claim should not.

\section{References}

1. Allred, S (2009) 'Reframing Asperger syndrome: lessons from other challenges to the Diagnostic and statistical manual and ICIDH approaches', Disability \& Society, 24: 3, 343 - 355.

2. Baker (2006) 'Neurodiversity, neurological disability and the public sector: notes on the autism spectrum', Disability \& Society, 21(1), 15- 29.

3. Baron-Cohen, S (2002) Is Asperger Syndrome Necessarily Viewed as a Disability? Focus on autism and other developmental disabilities, 17(3), 186-191.

4. Broderick, AA (2008) 'Autism as metaphor: narrative and counter-narrative', International Journal of Inclusive Education, 12(5), 459- 476.

5. Brownlow, C, O'Dell, L (2009) Challenging understandings of Theory of Mind: A brief report. Intellectual and developmental disabilities 47 (6): 473-478.

6. Davidson, J (2008) Autistic culture online: virtual communication and cultural expression on the spectrum. Social \& Cultural Geography 9 (7):791-806.

7. Farrugia, D (2009) Exploring stigma: medical knowledge and the stigmatisation of parents of children diagnosed with autism spectrum disorder. Sociology of Health \& Illness 31 (7) 1011-1027.

8. Fenton, A, Krahn, T (2007) Autism, Neurodiversity and Equality Beyond the 'Normal'. Journal of Ethics in Mental Health 2(2) 1-6.

9. Fitzgerald, M., O'Brien, B., (2007) Genius genes. how Asperger talents changed the world. Shawnee Mission/Autism Asperger Publishing Company

10. Goodin, R E (1985) Protecting the vulnerable: a reanalysis of our social responsibilities. Chicago: The University of Chicago Press.

11. Grandin, T (2006) Thinking in pictures and other reports from my life with autism. London: Bloomsbury.

12. Griffin, E, Pollak, D (2009) Student Experiences of Neurodiversity in Higher Education: Insights from the BRAINHE Project, Dyslexia 15: 23-41.

13. Jones, R.S.P., Zahl, A., Huws, J.C. (2001) First-hand Accounts of Emotional Experiences in Autism: a qualitative analysis. Disability \& Society, 16(3), 393-401.

14. Jurecic, A (2006) Mindblindness: Autism, Writing, and the Problem of Empathy, Literature and Medicine 25 (1): 1-23.

15. Kymlicka, W (1996) Multicultural Citizenship - A Liberal Theory of Minority Rights (p. 107-110) Oxford: Oxford University Press.

16. Lawson, W (2009) Single attention and associated cognition in autism (SAACA). PhD thesis Deakin University. 
18. McGeer, V (2004) Autistic self-awareness. Philosophy, Psychiatry \& Psychology 11(3): 253-251.

19. Moy, SS, Nadler, JJ (2008) Advances in behavioral genetics: mouse models of autism, Molecular Psychiatry, 13, 4-26

20. Muhle, R (2004) The genetics of autism. Pediatrics 113: e472-e486.

21. Nordenfelt, L (1987) On the nature of health. Dordrecht: Reidel.

22. Ortega, F (2009) The cerebral subject and the challenge of neurodiversity. Biosciences 4, 425-445.

23. Parens, E, Asch, A (2003) Disability rights critique of prenatal genetic testing: reflections and recommendations. Mental retardation and developmental disabilities 9: 40-47 (2003)

24. Ruof, M C (2004) Vulnerability, Vulnerable Populations, and Policy. Kennedy Institute of Ethics Journal, 14 (4), 411-425

25. Schroeder, D, Gefenas, E (2009) Vulnerability: Too Vague and Too Broad? Cambridge Quarterly of Healthcare Ethics , 18, 113-121.

26. Singer, J (1999) 'Why can't you be normal for once in your life?' From a 'problem with no name' to the emergence of a new category of difference. In: Disability Discourse, Corker, M and French, S (eds), Buckingham/Philadelphia: Open University Press.

27. Williams, D (2002) Exposure Anxiety--The Invisible Cage : An Exploration of Self-Protection Responses in the Autism Spectrum, Jessica Kingsley Publishers.

28. Wing, L (1997) The autistic spectrum. The Lancet, 350 (9093), 1761-1766

29. Aspies for freedom. Pro-neurodiversity website. Found at: http://www.aspiesforfreedom.com. Accessed 15/09/2010.

30. The human neurodiversity laboratory. Pro-neurodiversity website. Found at: http://eckertlab.org/neurodiversity, accessed 15/09/2010

31. Wolbring, G (2007) Neurodiversity, Neuroenhancement, Neurodisease, and Neurobusiness. Proneurodiverswity website. Found at: http://innovationwatch-archive.com/choiceisyours/choiceisyours2007-04-30.htm, accessed 15/09/2010.

\footnotetext{
32. Ventura33's Neurodiversity Page. Pro-neurodiversity website. Found at: http://www.ventura33.com/neurodiversity/, accessed 15/09/2010.

33. American Psychiatric Association: DSM-V development. Found at:
} 